\title{
Characterising gross plastic deformation in design by analysis
}

\author{
Hongjun Li*, Donald Mackenzie \\ Department of Mechanical Engineering, University of Strathclyde, 75 Montrose St., Glasgow G1 1XJ, UK
}

Received 20 January 2005; revised 12 April 2005; accepted 13 June 2005

\begin{abstract}
An investigation of three simple structures is conducted to identify and characterise the condition of gross plastic deformation in pressure vessel design by analysis. Limit analysis and bilinear hardening plastic analysis is performed for three simple example problems. It is found that previously proposed plastic criteria do not fully represent the effect of the hardening material model on the development of the plastic failure mechanism. A new criterion of plastic collapse based on the curvature of the load-plastic work history is therefore proposed. This is referred to as the Plastic Work Curvature or PWC criterion. It is shown that salient points of curvature correspond to critical stages in the physical evolution of the gross plastic deformation mechanism. The PWC criterion accounts for the effect of the bilinear hardening model on the development of the plastic mechanism and gives an enhanced plastic load when compared to the limit load.
\end{abstract}

(C) 2005 Elsevier Ltd. All rights reserved.

Keywords: Design by analysis; Gross plastic deformation; Plastic load; Criterion of plastic collapse

\section{Introduction}

Gross plastic deformation under static loading is a fundamental failure mode considered in pressure vessel design by analysis (DBA), as defined in Codes of Practice such as PD5500 Unfired Fusion Welded Pressure Vessels [1], EN13445: Part 3, Unfired Pressure Vessels [2] and ASME Boiler \& Pressure Vessel Code Section VIII Division 2 [3]. In elastic DBA, gross plastic deformation is prevented by limiting the primary stress in the vessel. In inelastic DBA, gross plastic deformation is prevented by limiting the load applied to the vessel, restricting it to a fraction of the notional ductile collapse load of the vessel. This paper considers how the ductile collapse load is characterised in inelastic DBA. In practice, this is done through limit analysis, which assumes an elastic-perfectly plastic material model, or by performing a more complex elasticplastic analysis which may include strain hardening and large deformation effects.

The Codes include different provisions and guidelines for inelastic analysis. Refs. [1-3] define procedures for

\footnotetext{
* Corresponding author. Fax: +44 1415525105.

E-mail address: hongjun.li.100@strath.ac.uk (H. Li).
}

0308-0161/\$ - see front matter (C) 2005 Elsevier Ltd. All rights reserved. doi:10.1016/j.ijpvp.2005.06.003 calculating the ductile collapse load of a vessel through conventional limit analysis. The limit load is the highest load satisfying equilibrium between external and internal forces and may be assumed to be the ductile collapse load in DBA. Limit analysis does not consider the effect large deformations have on the structural response and the equilibrium calculation is based on the initial geometry of the structure. Large deformation or non-linear geometry effects may enhance or diminish the load carrying capacity of the structure, referred to as geometric strengthening and geometric weakening, respectively. When large deformation effects are included in the analysis, the equilibrium calculation is based on the deformed geometry of the structure.

PD5500 does not refer to large deformation effects in inelastic DBA. EN13445 Annex B Design by AnalysisDirect Route [2] states "In checks on structures where deformation has an unfavourable (weakening) effect, geometrically non-linear effects shall be taken into account in gross plastic deformation checks". Thus, EN13445 requires that any geometric weakening effect is included in the analysis. In such a case, the calculated collapse load, referred to as a 'lower bound limit value', is not a conventional limit load. The equilibrium calculation is based on the deformed geometry of the structure and collapse may occur due to structural instability. EN13445 does not specify any guidelines for geometrical 


\begin{tabular}{|c|c|c|c|}
\hline \multicolumn{4}{|c|}{ Nomenclature } \\
\hline$a$ & inner radius of the cylinder & $V_{i}$ & volume of bar $i$ \\
\hline$b$ & width of the cantilever beam & $W_{\mathrm{E}}$ & elastic work \\
\hline$b_{\mathrm{c}}$ & outer radius of the cylinder & $W_{\mathrm{P}}$ & plastic work \\
\hline$d$ & depth of the cantilever beam & $\alpha$ & ratio of plastic work to elastic work at plastic \\
\hline$L$ & length of the cantilever beam & & load \\
\hline$L_{i}$ & length of bar $i$ & $\sigma_{i}$ & stress of bar $i$ \\
\hline E & Young's modulus & $\varepsilon_{i}^{\mathrm{el}}$ & elastic strain of bar $i$ \\
\hline$E_{\mathrm{pl}}$ & plastic modulus of the bilinear hardening & $\varepsilon_{i}^{\mathrm{pl}}$ & plastic strain of bar $i$ \\
\hline & material & $\theta$ & rotation of the cross-section of the beam \\
\hline$I$ & second moment of area & $\rho$ & radius of curvature \\
\hline
\end{tabular}

strengthening and should this occur the enhanced strength is ignored and the allowable load based on conventional limit analysis.

Large deformation effects may be included in the ASME VIII DBA procedure in what is termed a plastic analysis, which may also include material strain hardening (neither PD5500 nor EN13445 consider strain hardening). The ductile collapse load calculated in a plastic analysis is not the load required to cause physical collapse of the real vessel. It is the load at which gross plastic deformation occurs and is thus referred to as the 'plastic load' rather than plastic collapse load, as suggested by Gerdeen [4]. In ASME VIII, the plastic load is defined by applying the twice elastic slope (TES) criterion of plastic collapse to a characteristic loaddeformation curve for the vessel. In the TES criterion, the structural response is characterised by plotting a load parameter against a deformation parameter. A straight collapse limit line is then drawn from the origin of the characteristic curve with slope of half the stiffness of the initial elastic response, as shown in Fig. 1(a). (This is often referred to as 'twice the elastic slope' as in the ASME procedure the collapse limit line is defined in terms of the angle between the line and the load axis). The plastic load is defined as the load corresponding to the intersection of the collapse limit line and the load-deformation curve.

\section{Determining plastic load}

The ASME VIII Div 2 twice elastic slope (TES) criterion of plastic collapse is one of several similar criteria that have been proposed, some of which were incorporated in earlier versions of the ASME Code. The 1\% plastic strain method [5], the twice elastic deformation method [6], the $0.2 \%$ offset strain criterion [7], the proportional limit criterion [7] and the tangent-intersection (TI) criterion [8] all define the plastic load by applying a heuristic graphical constructions to characteristic load-deformation curves. In all cases, the calculated plastic load depends on the load and deformation parameters chosen to characterise the response.

Moffat et al. [9] investigated both the TES and TI methods for determining plastic loads for branch connections. In the TI criterion, two straight lines are drawn on the characteristic load-deformation curve, one tangent to the initial elastic response and one tangent to the plastic deformation region of the curve, as shown in Fig. 1(b). The load corresponding to the intersection of the two straight lines is defined as the plastic load. Moffat showed that the TES did not give a unique value for the plastic load of a branch due to the sensitivity of the criterion to the elastic response of the structure remote from the region where the plastic failure mechanism actually occurs. However, the TI method was found to give a unique value of plastic load, independent of the elastic behaviour, provided the characteristic load deformation curve exhibited a steady-state

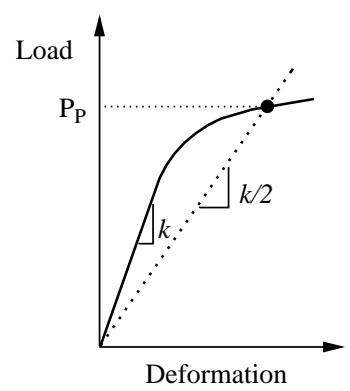

(a)

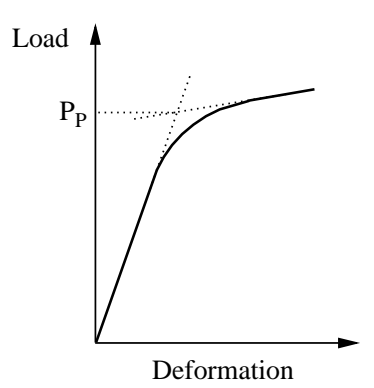

(b)

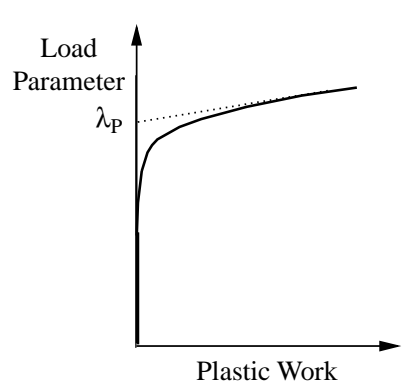

(c)

Fig. 1. DBA plastic criteria. (a) Twice elastic slope. (b) Tangent intersection. (c) Plastic work. 
response after the initial plastic zones formed. This was confirmed by Muscat et al. [10]. Robertson and the present writers investigated the evolution of plastic failure mechanisms in pipe bends under combined pressure and closing in-plane moment loading and applied both the TES and TI criteria to define plastic loads [11]. It was found that the calculated plastic load was dependent on the criterion used and how that criterion was interpreted. In the case of the TI criterion, choosing the location at which to draw the tangent to the plastic deformation part of the loading curve is subjective and significant variation in the calculated plastic pressure is possible.

Gerdeen attempted to provide a more rigorous justification for choice of plastic load by considering the relationship between work done on the vessel by the external loads and the plastic work dissipated in the vessel as load is increased [4]. He postulated that the plastic load occurs when the plastic work $W_{\mathrm{P}}$ is a specific factor $\alpha$ of the elastic work $W_{\mathrm{E}}$. Gerdeen did not define a general value of $\alpha$ indicating gross plastic deformation but showed that for certain configurations, certain values of $\alpha$ gave a correspondence with previous criteria such as the TES criterion. Limitations in inelastic analysis methods at the time led Gerdeen to characterise the elastic and plastic work in terms of areas under characteristic loaddeformation curves, similar to those used in methods such as the TES criterion. Consequently, his approach was dependent on the choice of local load and local deformation parameters. Muscat et al. [10] proposed a plastic collapse criterion based on a characteristic plot of a global load parameter (representing all applied loads) against the total or global plastic work in the vessel calculated by inelastic finite element analysis. In the plastic work (PW) criterion it is not necessary to define load or deformation parameters, which is convenient when multiple loads are applied. The characteristic curve in the PW criterion characterises the global response of the vessel. However, the criterion is somewhat arbitrary in that the designer is required to judge when the plastic work becomes excessive. Muscat defined the plastic load as the intersection between a straight-line tangent from the 'steady-state' region of the characteristic curve and the vertical axis, as shown in Fig. 1(c) (If there is no steadystate, it is suggested that a point on the curve corresponding to a maximum principal strain of $5 \%$ be used). Lee [12] has also proposed a plastic criterion based on plastic work concepts, specifically for cracked cylinder problems. The criterion is based on the concept that the plastic work dissipated varies in proportion with certain geometry parameters. The plastic load is defined as the load when the plastic work dissipated in the cracked cylinder is equal to the limit state plastic work of an uncracked cylinder factored by the geometry parameters.

The objective of this paper is to investigate how gross plastic deformation and plastic collapse can be characterised in terms of plastic work concepts for pressure vessel design by analysis. The evolution of plastic failure mechanisms in real pressure vessel configurations is usually a complex process, influenced by load history, material model and geometric features. The load-deformation and load-plastic work curves for these structures may have a complex form, making it difficult to identify specific characteristics of collapse. The present investigation therefore considers three simple structural configurations in which distinct characteristics of plastic collapse can be clearly identified.

\section{Plastic analysis of simple configurations}

Three simple models are considered: a three-bar system under axial force, a cantilever beam under a tip bending moment and a thick cylinder under internal pressure. Two material models are considered: elastic-perfectly plastic and bilinear hardening. All structures were analysed using the ANSYs [13] program, both for small and large deformation theory. Closed form analytical solutions for the elastic-plastic response of the three bar structure and the beam (assuming small deformation theory) obtained by $\mathrm{Li}$ [14] are also presented.

\subsection{Three bar structure}

The first simple structure is a system of three parallel bars of equal cross-section $A$ and different lengths $L$, such that $L_{3}>L_{2}>L_{1}$, as shown in Fig. 2. The bars are fixed at one end and constrained to equivalent axial deformation $d$ at the other when force $F$ is applied.

In an elastic analysis, the highest stress occurs in bar 1 and the lowest stress in bar 3. As $F$ increases, bar 1 yields first, then bar 2 and then bar 3. Assuming a bilinear strain hardening material model, the load-deformation response of the structure is given by

Wholly elastic:

$$
F=\operatorname{EAd}\left(\frac{1}{L_{1}}+\frac{1}{L_{2}}+\frac{1}{L_{3}}\right)
$$

Bar 1 plastic:

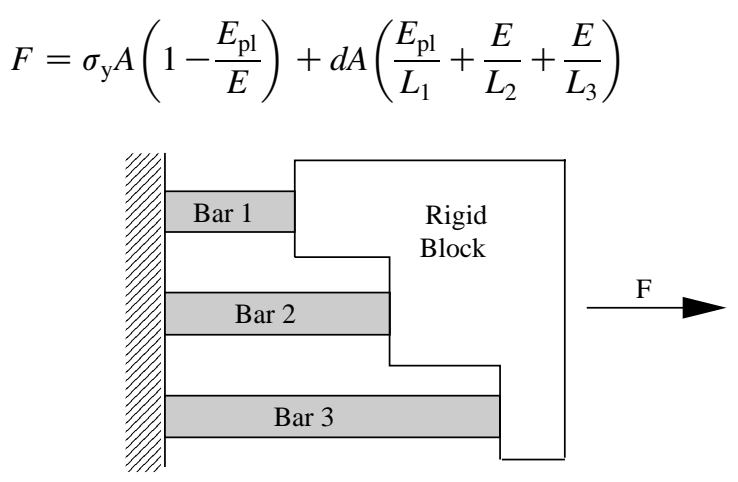

Fig. 2. Three bar structure. 
Bars 1 and 2 plastic:

$F=2 \sigma_{\mathrm{y}} A\left(1-\frac{E_{\mathrm{pl}}}{E}\right)+d A\left(\frac{E_{\mathrm{pl}}}{L_{1}}+\frac{E_{\mathrm{pl}}}{L_{2}}+\frac{E}{L_{3}}\right)$

Fully plastic:

$F=3 \sigma_{\mathrm{y}} A\left(1-\frac{E_{\mathrm{pl}}}{E}\right)+E_{\mathrm{pl}} d A\left(\frac{1}{L_{1}}+\frac{1}{L_{2}}+\frac{1}{L_{3}}\right)$

where $\sigma_{\mathrm{y}}$ is the yield stress, $E$ is the elastic modulus and $E_{\mathrm{pl}}$ the tangent modulus of the bilinear hardening material. The elastic and plastic work done on the structure as the load increases are obtained from uniaxial stress and strain theory. The elastic work $W_{\mathrm{E}}$ is

$W_{\mathrm{E}}=\frac{1}{2} \sum_{i=1}^{3} \sigma_{i} \varepsilon_{i}^{\mathrm{el}} V_{i}$

where $\sigma_{i}$ is the stress, $\varepsilon_{i}^{\mathrm{el}}=\sigma_{i} / E$ is the elastic strain and $V_{i}$ is the volume of bar $i$. The plastic work is calculated from

$W_{\mathrm{P}}=\sum_{i=1}^{3} \sigma_{i}^{m} \varepsilon_{i}^{\mathrm{pl}} V_{i}$

where $\sigma_{i}^{m}$ is the average stress in bar $i$

$\sigma_{i}^{m}=\frac{\sigma_{i}+\sigma_{\mathrm{y}}}{2}$

and $\varepsilon_{i}^{\mathrm{pl}}=\varepsilon_{i}-\varepsilon_{i}^{\mathrm{e}}$ is the plastic strain.

The elastic-plastic response of the general three bar structure is characterised in terms of load-deformation behaviour and load-plastic work behaviour by the analytical solution of Eqs. (1)-(7). Here, the response of a specific structure with dimensions $A=40 \mathrm{~mm}^{2}, L_{1}=40 \mathrm{~mm}, L_{2}=$ $80 \mathrm{~mm}, L_{3}=120 \mathrm{~mm}$ and material properties $E=200 \mathrm{GPa}$, yield stress $\sigma_{\mathrm{y}}=300 \mathrm{MPa}$ and $E_{\mathrm{pl}}=0 \mathrm{GPa}$ or $E_{\mathrm{pl}}=4 \mathrm{GPa}$ is considered.

Force-displacement plots for the elastic-perfectly plastic and bilinear hardening materials are presented in Fig. 3(a) and (b), respectively. Both curves show distinct changes in

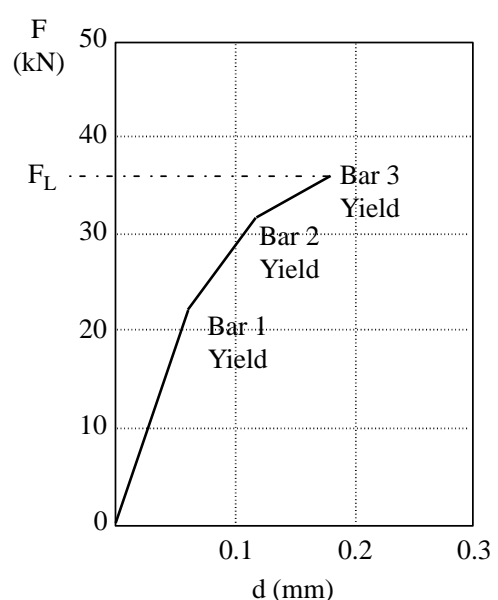

(a) slope as the bars yield in sequence 1, 2, 3. In the elasticperfectly plastic structure, the limit load is reached when bar 3 yields, at $F_{\mathrm{L}}=36 \mathrm{kN}$. Above this load, equilibrium between the internal and external forces is violated and unlimited plastic flow occurs. In the strain hardening structure, the structure becomes fully plastic when bar 3 yields at $F_{\mathrm{FP}}=39 \mathrm{kN}$ but the structure can continue to support increasing load indefinitely, due to the bilinear hardening material model, and a criterion of plastic collapse must be used to define the plastic load. Applying the TES and TI criteria to the strain-hardening force-deformation curve of Fig. 3(b) gives plastic loads $F_{\mathrm{TES}}=41 \mathrm{kN}$ and $F_{\mathrm{TI}}=36 \mathrm{kN}$.

The evolution of gross plastic deformation in the three bar structures is characterised in terms of plastic work dissipation in two plots in Fig. 4. Fig. 4(a) is a plot of applied load against plastic work dissipation, as used in Muscat's PW criterion. Fig. 4(b) is a plot of applied load against the ratio of plastic work dissipated, $W_{\mathrm{P}}$, to elastic strain energy stored, $W_{\mathrm{E}}$. Gerdeen proposed that the load corresponding to a specific value of the ratio $\alpha=W_{\mathrm{P}} / W_{\mathrm{E}}$ could characterise the plastic load but did not define a general value for $\alpha$.

Fig. 4(a) shows that the elastic-perfectly plastic structure experiences more plastic dissipation for a given (post-yield) load than the strain hardening structure (as bar 1 can continue to store part of the external work done as elastic strain energy). As load is increased, bars 2 and 3 of the strain hardening structure remain elastic at loads greater than the corresponding yield loads for these bars in the elasticperfectly plastic structure. The elastic-perfectly plastic structure becomes fully plastic when the limit load $F_{\mathrm{L}}=$ $36 \mathrm{kN}$ is reached. Thereafter, the theoretical plastic dissipation increases without bound as unlimited plastic flow occurs. The strain hardening structure becomes fully plastic when the applied load reaches $F_{\mathrm{FP}}=39 \mathrm{kN}$ but it continues to support increasing load. The rate of plastic dissipation is proportional to the increase in load and, in accordance with the material model, it can continue to

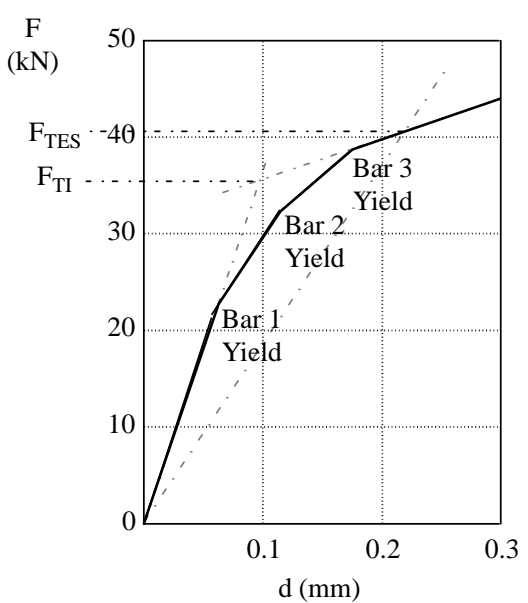

(b)

Fig. 3. Three bar structure force-displacement plots: (a) elastic-perfectly plastic material and (b) bilinear hardening material. 


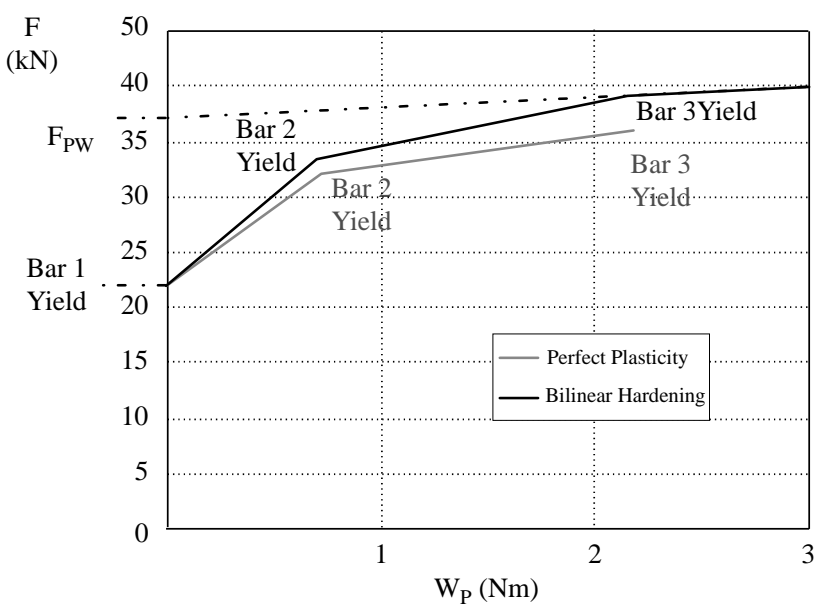

(a)

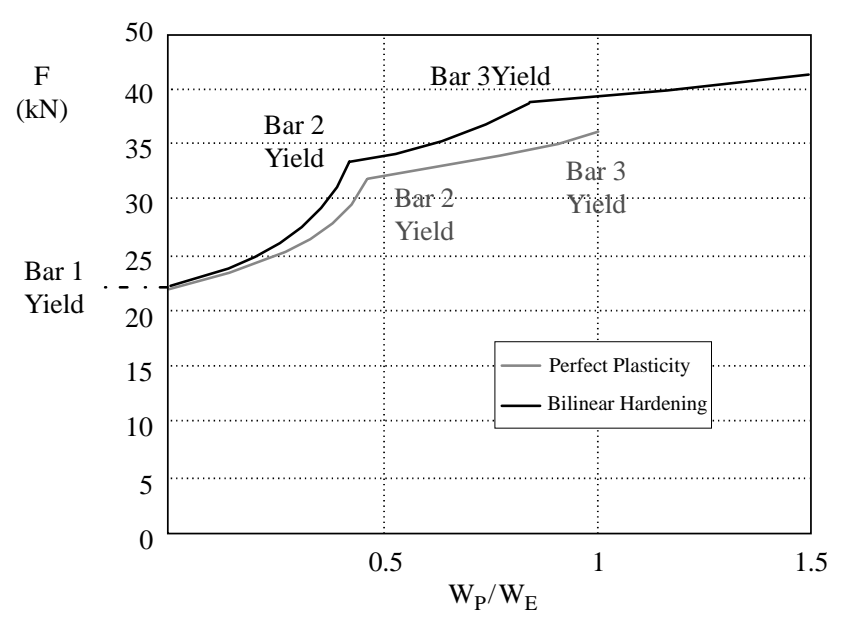

(b)

Fig. 4. Three bar structure: (a) force-plastic work and (b) force-work ratio (plastic to elastic) plots.

increase indefinitely. Applying Muscat's PW criterion [10] to the strain hardening curve gives a plastic load $F_{\mathrm{PW}}=37.5 \mathrm{kN}$.

The force- $W_{\mathrm{P}} / W_{\mathrm{E}}$ curves for elastic-perfectly plastic and strain hardening materials in Fig. 4(b) show distinct changes in response at points corresponding to yield of each bar, indicating distinct changes in elastic-plastic behaviour. For a given load, $W_{\mathrm{P}} / W_{\mathrm{E}}$ is greater for the elastic-perfectly plastic material than for the strain hardening material. Considering the elastic-perfectly plastic material, the ratio $W_{\mathrm{P}} / W_{\mathrm{E}}$ at the limit load has a $\alpha_{\mathrm{L}}=1$. At the fully plastic load in the strain hardening structure, the ratio has value $\alpha_{\mathrm{FP}}=$ 0.84 .

\subsection{Beam in bending}

The second structure considered is a rectangular cantilever beam width $b$, depth $d$ and length $L$ subject to a pure bending moment $M$, as shown in Fig. 5(a). Closed form expressions for moment-curvature and moment-work

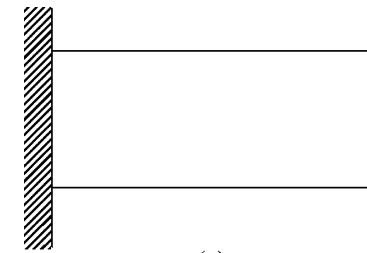

(a)

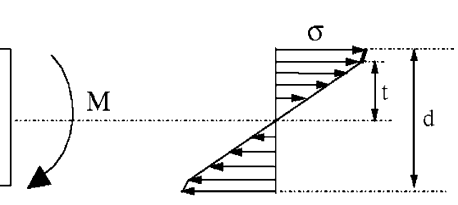

(b)
Fig. 5. Cantilever beam subject to end bending moment.

relationships for elastic and elastic-plastic deformation were obtained by Li [14], assuming Engineers' bending theory and small deformations. When large deformations are included, the analysis becomes more complex and large deformation analysis was performed by the Finite Element Method.

The moment $M$-rotation $\theta$ relationship in the elastic range is

$M=\frac{E I \theta}{L}$

where $E$ is the Young's modulus and $I$, the second moment of area. Assuming a bilinear strain hardening material, the distribution of stress through the cross-section in the elasticplastic range is shown in Fig. 5(b). The half-depth of the elastic core of the beam is denoted $t$. The applied moment $M$ and half-depth of the elastic zone $t$ are related by the equation

$M=\frac{b d^{2} \sigma_{\mathrm{y}}}{4}\left(1-\frac{E_{\mathrm{pl}}}{E}\right)+\frac{b d^{3} E_{\mathrm{pl}} \sigma_{\mathrm{y}}}{12 E t}-\frac{1}{3} b \sigma_{\mathrm{y}} t^{2}\left(1-\frac{E_{\mathrm{pl}}}{E}\right)$

This shows that in a bilinear hardening material, the moment tends to infinity as the yield front approaches the neutral surface, $t \rightarrow 0$. The relationship between the applied moment and rotation of the cross-section $\theta$ is:

$M=\frac{b d^{2} \sigma_{\mathrm{y}}}{4}\left(1-\frac{E_{\mathrm{pl}}}{E}\right)+\frac{b d^{3} E_{\mathrm{pl}} \theta}{12 L}-\frac{b \sigma_{\mathrm{y}}^{3} L^{2}}{3 E^{2} \theta^{2}}\left(1-\frac{E_{\mathrm{pl}}}{E}\right)$

Eq. (10) shows that when the rotation $\theta$ is large, the relationship between moment and rotation is approximately linear, with slope $K_{\mathrm{p}}$ given by

$K_{\mathrm{p}}=\frac{b d^{3} E_{\mathrm{pl}}}{12 L}$

Finite element analysis was used to investigate the development of the plastic failure mechanism for a beam of specific dimensions $b=10 \mathrm{~mm}, d=10 \mathrm{~mm}, L=50 \mathrm{~mm}$, $E=200 \mathrm{GPa}, E_{\mathrm{pl}}=4 \mathrm{GPa}, \sigma_{\mathrm{y}}=300 \mathrm{MPa}$, and $\nu=0$. The beam was fixed at one end and a bending moment applied to the free end. Moment rotation plots for the elastic-perfectly plastic and bilinear hardening materials are presented in Fig. 6(a) and (b), respectively. There is precise agreement between the analytical and finite element results for small deformation analysis. The curves reach a linear steady-state 


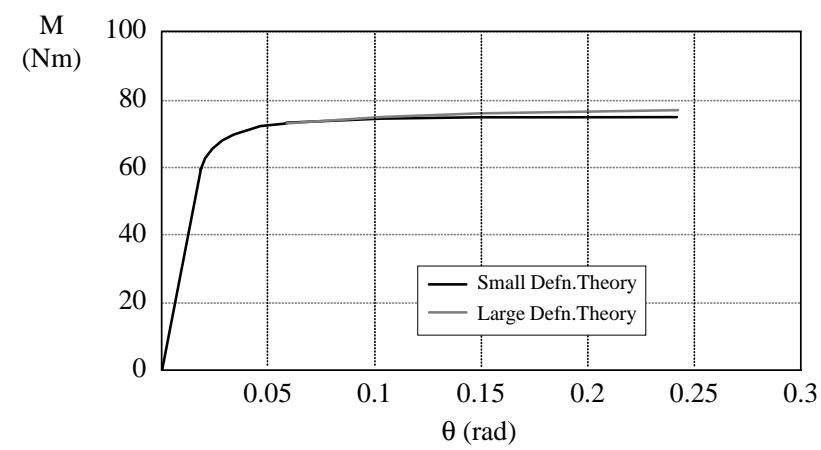

(a)

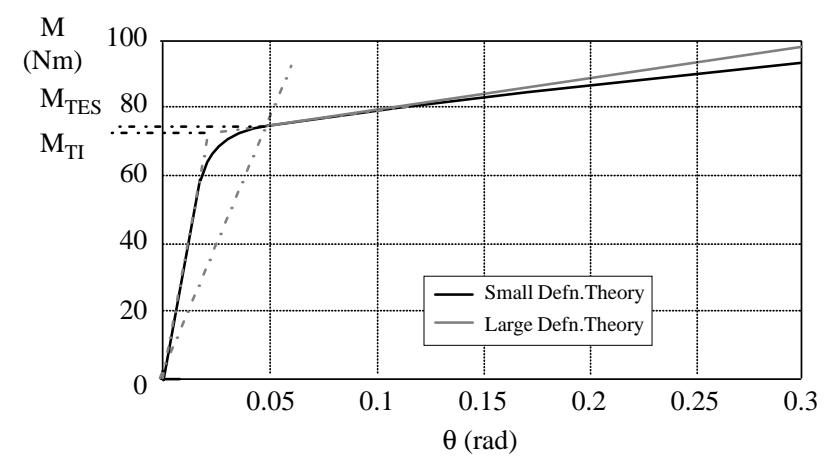

(b)

Fig. 6. Beam moment-rotation plots: (a) elastic-perfectly plastic material and (b) bilinear hardening material.

for rotations $\theta>0.1 \mathrm{rad}$. The slope of the steady-state portions of the curves are consistent with Eq. (11): zero for an elastic-perfectly plastic material and $67 \mathrm{Nm} / \mathrm{rad}$ for bilinear hardening. The form of the curves is different to those for the three bar structure, Fig. 3, which exhibited distinct changes in slope as each bar yielded in turn. In the beam, the transition from elastic to plastic behaviour is less clearly defined and the post-yield response shows a smooth transition from the initial elastic slope to the steady-state plastic slope.

The applied moment is plotted against the depth of the plastic zones, expressed as a percentage of the beam depth, for the elastic-perfectly plastic and bilinear hardening materials in Fig. 7. As the load is increased, the plastic zones grow at approximately the same rate until the applied moment approaches the limit moment. As the limit load is reached in the elastic-perfectly plastic structure, the plastic zones meet to form the limit collapse mechanism. The corresponding load is the limit load of the structure. In the hardening structure, the two plastic zones approach the midsurface asymptotically with respect to applied moment. Theoretically, Eq. (9), they do not meet for any value of moment and the section never becomes fully plastic.

Analytical expressions for elastic work and plastic work in an elastic-perfectly plastic beam were obtained by substituting the through-depth variations in stress and strain into Eqs. (5) and (6) and integrating through the volume of the beam. In the elastic range, the work done on the beam,

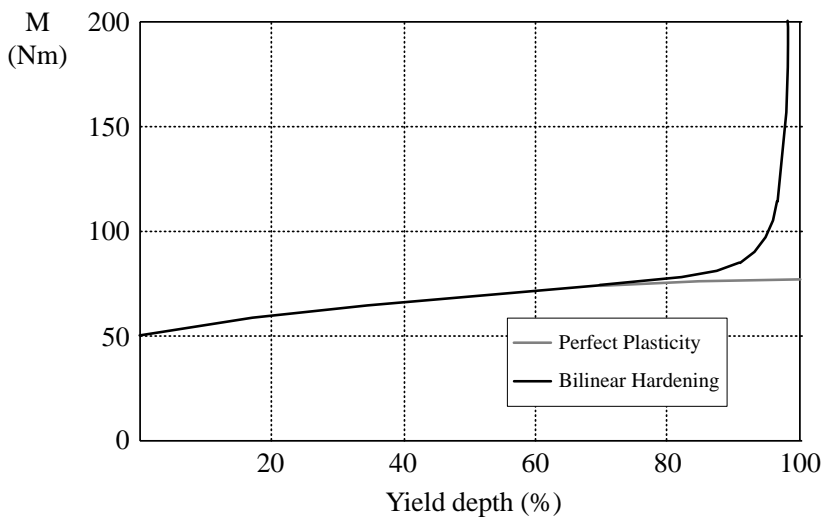

Fig. 7. Variation in depth of plastic zone with applied moment.

$W$, is:

$W=\frac{E I \theta^{2}}{2 L}$

The post-yield elastic work is given by

$W_{\mathrm{E}}=\frac{b \mathrm{~d} L \sigma_{\mathrm{y}}^{2}}{2 E}-\frac{2 b L^{2} \sigma_{\mathrm{y}}^{3}}{3 E^{2} \theta}$

and the plastic work is

$W_{\mathrm{P}}=b L \sigma_{\mathrm{y}}\left(\frac{\theta d^{2}}{4 L}+\frac{L \sigma_{\mathrm{y}}^{2}}{E^{2} \theta}-\frac{\sigma_{\mathrm{y}} d}{E}\right)$

Characteristic plastic work plots for the beam are shown in Fig. 8. The small and large deformation theory results are similar for the elastic-perfect plastic material and only the plots for small deformation theory are shown. Fig. 8(a) is a load-plastic work plot and Fig. 8(b) is a plot of the applied moment against the ratio of plastic work to elastic work. Both plots show a smooth transition from elastic to plastic response. As in the 3-Bar structure, after initial yield the amount of plastic dissipation in the elastic-perfectly plastic beam is greater than in the hardening structure for any given load, as the plastic zones spreading from the top and bottom surfaces can continue to store part of the total work done as elastic strain energy.

The limit moment of the beam, the last converged equilibrium solution in limit analysis, is $M_{\mathrm{L}}=75 \mathrm{Nm}$. Plastic moments were calculated by applying the TES and TI criteria to the moment-rotation plots of Fig. 6(b), giving values of plastic load just below the limit load: $M_{\mathrm{TES}}=$ $74 \mathrm{Nm}$ and $M_{\mathrm{TI}}=72 \mathrm{Nm}$, respectively. Applying Muscat's PW criterion to Fig. 8(a) gives a slightly higher plastic load, $M_{\mathrm{PW}}=78 \mathrm{Nm}$. All three criteria therefore give values of plastic load similar to the calculated limit load, indicating that the criteria do not capture the effect of the hardening material model on the post-yield stress redistribution. Compared to an elastic-perfectly plastic material, bilinear hardening impedes the spread of plastic deformation and a higher load is therefore required to cause gross plastic deformation. Referring to Fig. 7, it is seen that at 


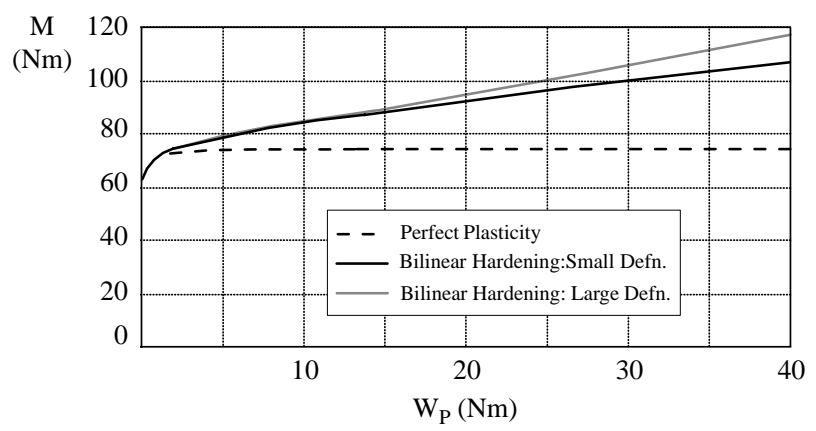

(a)

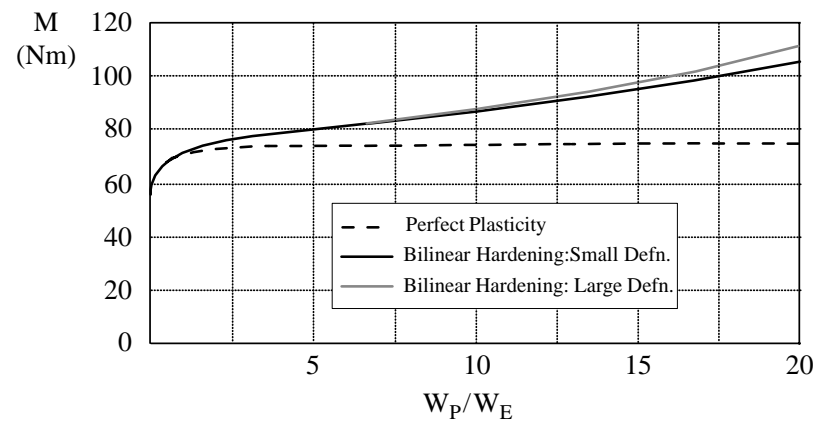

(b)

Fig. 8. Beam (a) force-plastic work and (b) force-work ratio (plastic to elastic) plots.

the calculated plastic loads approximately $50 \%$ of the crosssection of the hardening beam has deformed plastically, compared to $100 \%$ in the elastic-perfectly plastic beam. Clearly, there is a quantitative difference in the implicit definition of 'gross plastic deformation' between the limit and plastic DBA procedures when the TES, TI, and PW criteria are applied to the beam.

The plot of the applied moment against the ratio of plastic work to elastic work, Fig. 8(b), indicates that the $\alpha$ value increases without limit as the limit load is approached, (also seen in the ratio of Eqs. (13) and (14) for large rotations $\theta$. Theoretically, $\alpha_{\mathrm{L}}=\infty$ at the limit load, as unlimited plastic work is done. The bilinear hardening solution also shows that the beam never achieves a fully plastic state and thus the value of $\alpha_{\mathrm{FP}}$ is again unlimited. This behaviour differs significantly from that found in the bar structure, for which distinct values of $\alpha_{\mathrm{L}}=1$ and $\alpha_{\mathrm{FP}}=$ 0.84 were identified. This result indicates that the value of $\alpha$ associated with plastic collapse is problem dependent and does not provide the basis for a general plastic criterion.

The results of the beam analysis show that the previously proposed plastic criteria do not define a problem-independent plastic load consistent with the Code concept of gross plastic deformation when compared with the limit analysis. However, a more consistent definition of gross plastic deformation and hence plastic load can be established by considering the form of the moment-plastic work plot used in the PW criterion, Fig. 8(a), in more detail. Fig. 8(a) presents the structural response in the conventional DBA format, with the deformation parameter (or plastic work) on the $x$-axis and the load on the $y$-axis (similar to the convention used in deformation-controlled material tensile tests, in which strain is plotted on the $x$-axis and stress on the $y$-axis). However, gross plastic deformation is by definition a load-controlled mechanism and the associated plastic work is a function of the applied load. It is therefore useful to plot the plastic work $W_{\mathrm{P}}$ against applied load $Q$, as illustrated schematically in Fig. 9. The slope at any point on the plastic work-load curve, $\mathrm{d} W_{\mathrm{P}} / \mathrm{d} Q$, is the rate of change of plastic deformation with increasing load. The rate of change of slope, $\mathrm{d}^{2} W_{\mathrm{P}} / \mathrm{d} Q^{2}$, characterizes how rapidly the rate of plastic deformation is changing with increasing load. The evolution of the gross plastic deformation mechanism can be characterised by considering the rate of change of slope of the $W_{\mathrm{P}}-Q$ curve. Alternatively, the response can be characterised by the curvature at a point on the curve. The curvature, the inverse of the radius of curvature $\rho$, is perhaps easier to visualise and is related to the rate of change of slope through the expression:

$$
\frac{1}{\rho}=\frac{\frac{\mathrm{d}^{2} W_{\mathrm{P}}}{\mathrm{d} Q^{2}}}{\left[1+\left(\frac{\mathrm{d} W_{\mathrm{P}}}{\mathrm{d} Q}\right)^{2}\right]^{3 / 2}}
$$

In a structure exhibiting an elastic-plastic response characterised by a load-plastic work curve such as that shown in Fig. 9, the initial elastic response has zero curvature. After yielding, stress redistribution occurs and the $W_{\mathrm{P}}-Q$ curve becomes non-linear. This is characterised by an increase in curvature to a maximum as the plastic deformation mechanism develops. The maximum rate of plastic stress redistribution occurs at the load corresponding to the maximum curvature. Thereafter, the curvature decreases, indicating decreasing stress redistribution, until an approximately constant minimum or zero value of curvature occurs, depending on the particular configuration. After this, little or no further stress redistribution occurs unless a second plastic deformation mechanism is initiated and the structure exhibits approximately

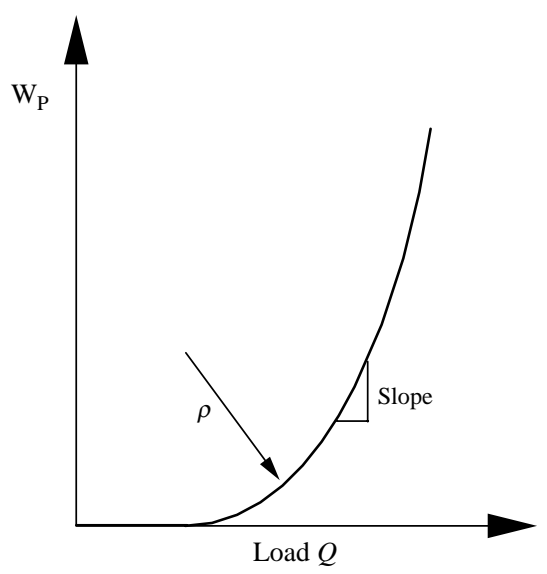

Fig. 9. Typical plot of plastic work against load. 
constant, gross plastic deformation. It is proposed that the load corresponding to this either constant or zero curvature is the Plastic Work Curvature or PWC criterion plastic load.

As the PWC criterion considers the curvature of the $W_{\mathrm{P}}-Q$ curve, it does not matter whether work is plotted against load or vice-versa when characterising the response. However, to conform to conventional practice, it is proposed that the plastic work be plotted on the $x$-axis and the load on the $y$-axis. The curvature of the load-plastic work curve can be obtained in several ways. Here, for convenience, the commercial modelling program Pro/Engineer [15] was used to evaluate the curvature. A cubic spline fit through load-plastic work data points was generated in ProE and the ProE Curvature function used to graphically display the curvature. Moment-plastic work plots for the limit and bilinear hardening analyses of the beam are shown in Fig. 10. The relative magnitude of the curvature is superimposed on the moment-plastic work curve (the normal distance from the curve to the superimposed curvature plot).

In the elastic-perfectly plastic beam, Fig. 10(a), the curvature increases from zero at yield moment $M_{\mathrm{y}}=50 \mathrm{Nm}$ to a maximum value before decreasing to zero at the PWC plastic load, $M_{\mathrm{PWC}}=75 \mathrm{Nm}$, the limit load of the beam. In the bilinear hardening model, the curvature initially increases slowly from zero at first yield before increasing rapidly to a maximum value at $75 \mathrm{Nm}$. The curvature then decreases rapidly to a discontinuity at $M=80 \mathrm{Nm}$, followed by a more gradual decrease to a second discontinuity in curvature at $M=87 \mathrm{Nm}$. Thereafter, the curvature has an approximately constant value and the moment $M_{\mathrm{PWC}}=$ $87 \mathrm{Nm}$ is defined as the PWC criterion plastic load. At this load, $90 \%$ of the beam cross-section has experienced plastic deformation (the bilinear hardening beam approaches the fully plastic state asymptotically, hence the curvature never actually reaches zero).

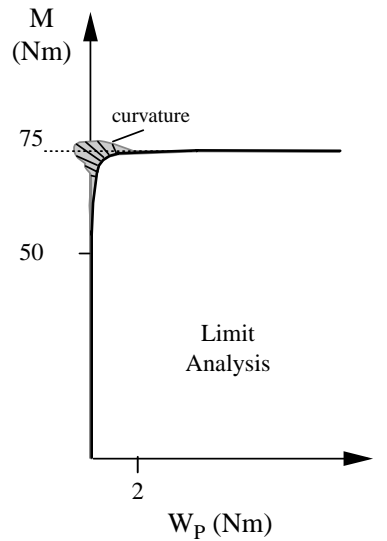

(a)

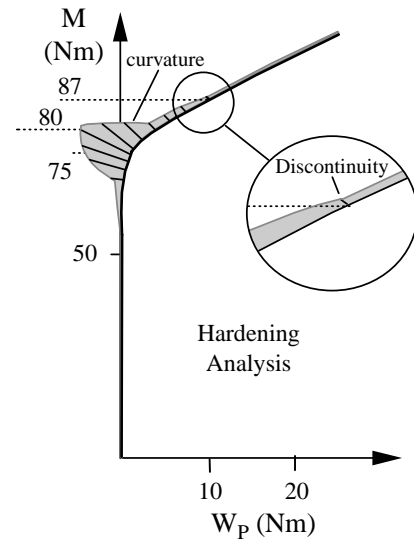

(b)
Fig. 10. Beam moment-plastic work plots with curvature superimposed (a) elastic-perfectly plastic material (b) bilinear hardening material.

\subsection{Thick cylinder under internal pressure}

The elastic load-deformation response of a thick cylinder under internal pressure assuming the Tresca yield criterion and small deformation theory is well known and documented in standard texts such as Lubliner [16]. Lee [12] has presented an analytical solution for the plastic work at the limit pressure of an elastic-perfectly plastic thick cylinder but no solution is available for the variation in plastic work with load (from first yield to limit state). Here, the thick cylinder example was analysed by FEA only. Small deformation and large deformation theories were considered and were found to give similar results. Only the small deformation theory results are presented here.

A $15^{\circ}$ segment of an open ended cylinder with inner radius $a=10 \mathrm{~mm}$, outer radius $b_{\mathrm{c}}=15 \mathrm{~mm}$ and length $L_{\mathrm{c}}=$ $4 \mathrm{~mm}$ was created. The material properties are $E=200 \mathrm{GPa}$, $E_{\mathrm{pl}}=4 \mathrm{GPa}, \sigma_{\mathrm{y}}=300 \mathrm{MPa}$, and $\nu=0.3$. Pressure is plotted against radial deformation (at the bore), assuming small deformation theory, for the elastic-perfectly plastic and bilinear hardening materials in Fig. 11. As in the beam example, the cylinder shows a smooth transition from elastic to plastic deformation. Initial yield occurs at the bore when $P_{\mathrm{y}}=94 \mathrm{MPa}$ and the plastic zone spreads out towards the outer surface as load is increased. In the elasticperfectly plastic cylinder, limit collapse occurs when the plastic zone spreads across the entire section. The structure is then fully plastic and cannot support further increase in pressure without violating equilibrium. The limit pressure of the cylinder, the last converged equilibrium solution in limit analysis, is $P_{\mathrm{L}}=131 \mathrm{MPa}$. In the strain hardening cylinder, greater post-yield pressures are required to extend the plastic zone a similar amount, as the plastic zone can store part of the work done as elastic strain energy. The plastic zone reaches the outer surface at pressure $P_{\mathrm{FP}}=133 \mathrm{MPa}$ and the cylinder is fully plastic. However, because of the bilinear hardening, the model can continue to support increasing pressure indefinitely and a criterion of plastic collapse is needed to define the plastic load. Plastic pressures were calculated by applying the TES and TI criteria to the pressure-displacement of Fig. 11, giving

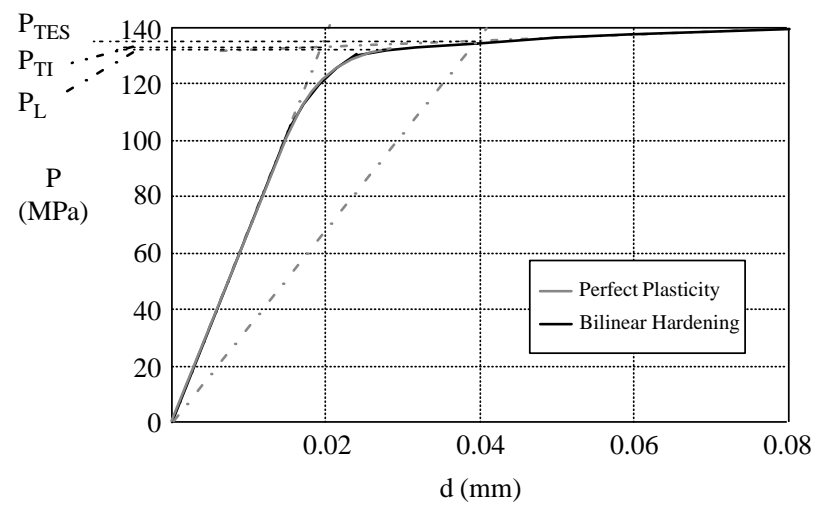

Fig. 11. Cylinder pressure-deformation (radial at bore) plot. 
$P_{\mathrm{TES}}=134 \mathrm{MPa}$ and $P_{\mathrm{TI}}=132 \mathrm{MPa}$. Therefore, in the cylinder example, the TES and TI criteria give a value of plastic load very close to the fully plastic load of the component.

The response of the cylinder is characterised in terms of plastic work in Fig. 12. Fig. 12(a) shows pressure against plastic work and Fig. 12(b) shows pressure against the ratio of plastic work to elastic work Applying Muscat's PW criterion to Fig. 12(a) gives a plastic load of $P_{\mathrm{PW}}=$ $133 \mathrm{MPa}$, equal to the fully plastic pressure. Considering Gerdeen's criterion, Fig. 12(b) shows that the $\alpha$ value at the limit pressure is $\alpha_{\mathrm{L}}=0.75$. The value at the fully plastic pressure of the strain hardening cylinder is $\alpha_{\mathrm{FP}}=1.6$.

The curvature of the pressure-plastic work curves for the cylinder is shown for the elastic-perfectly plastic and strain hardening material models in Fig. 13(a) and (b). With the elastic-perfectly plastic material model, the curvature increases to a maximum in the transition between elastic and plastic dominance and falls rapidly to zero at the limit load. In the bilinear hardening material, the curvature reaches a maximum at a pressure of $131 \mathrm{MPa}$. It then falls to a discontinuity in curvature at $133 \mathrm{MPa}$, which is the fully plastic load of the cylinder. Thereafter, the curvature gradually reduces to zero at pressure $P_{\mathrm{PWC}}=134 \mathrm{MPa}$.

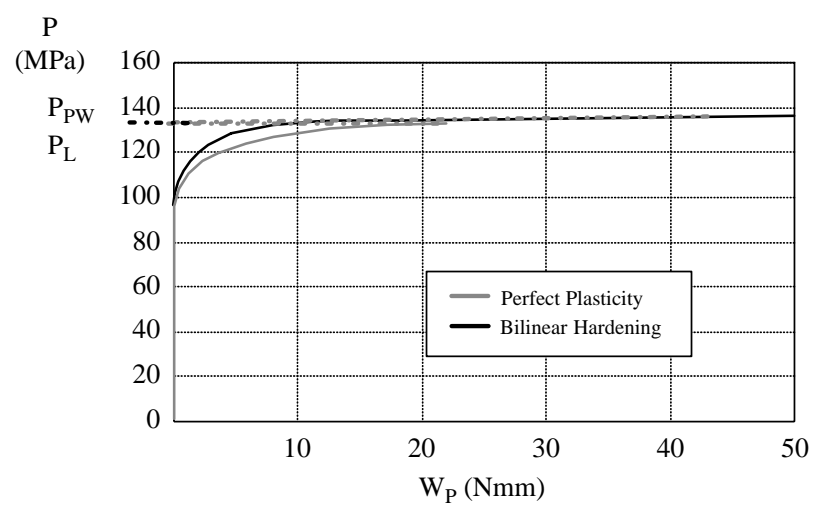

(a)

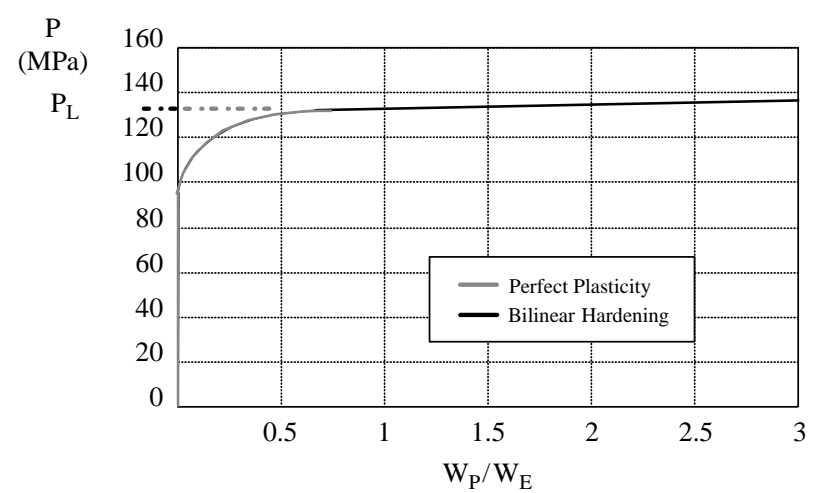

(b)

Fig. 12. Cylinder (a) pressure-plastic work and (b) pressure-work ratio (plastic to elastic) plots.

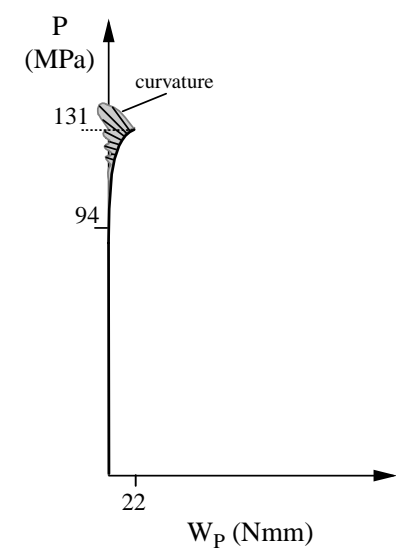

(a)

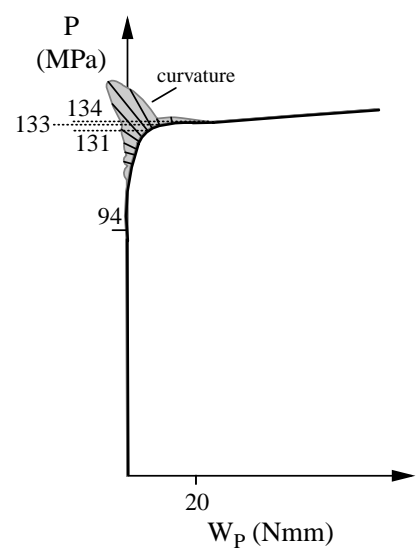

(b)
Fig. 13. Cylinder pressure-plastic work plots with curvature superimposed (a) elastic-perfectly plastic material and (b) bilinear hardening material.

\section{Discussion}

The simple examples considered identify features of gross plastic deformation in strain hardening structures that do not exhibit geometric weakening. When an elasticperfectly plastic material model is assumed, limit collapse of all three structures occurs when the load caused plastic deformation throughout the complete volume of the structure. When a bilinear hardening material model is assumed, the 3-bar structure and cylinder both eventually experience fully plastic deformation with increasing load but the beam structure approaches full plasticity asymptotically. Limit loads were determined for the bar and beam structure analytically and using FEA. Only FEA was used in the cylinder example. Plastic loads were defined by applying the established TES, TI, and PW criteria and the proposed PWC criterion. The load corresponding to fully plastic deformation was also calculated for the strain hardening 3-bar structure and cylinder. The results of the analyses are summarised in Table 1.

Table 1 shows that the limit and fully plastic loads for the bar structure and cylinder are relatively close. This indicates that including the strain hardening has little restraining effect on the spread of plasticity in these structures. In effect, there is little post-yield stress redistribution in these components and the TES, TI, and PW criteria all give similar values of plastic load. The TI and PW criteria give

Table 1

Comparison of plastic loads

\begin{tabular}{lccccccc}
\hline Load & Yield & Limit & $\begin{array}{l}\text { Fully } \\
\text { plastic }\end{array}$ & TES & TI & PW & PWC \\
\hline $\begin{array}{l}\text { 3-Bar structure } \\
\text { force (N) }\end{array}$ & 22.5 & 36 & 39 & 41 & 36 & 37.5 & 39 \\
$\begin{array}{l}\text { Beam moment } \\
(\mathrm{Nm})\end{array}$ & 50 & 75 & - & 74 & 72 & 78 & 87 \\
$\begin{array}{l}\text { Cylinder } \\
\text { pressure (MPa) }\end{array}$ & 94 & 131 & 133 & 134 & 132 & 133 & 134 \\
\hline
\end{tabular}


Table 2

Comparison of $\alpha$ factors at limit and fully plastic states

\begin{tabular}{lll}
\hline Factor & $\alpha_{\mathrm{L}}$ & $\alpha_{\mathrm{FP}}$ \\
\hline 3-Bar structure & 1.00 & 0.84 \\
Beam & $\infty$ & $\infty$ \\
Cylinder & 0.74 & 1.00 \\
\hline
\end{tabular}

values between the limit load and fully plastic load in both cases. The TES criterion gives a plastic load greater than the fully plastic load for the bar structure and a plastic pressure marginally greater than the fully plastic pressure for the cylinder. The new PWC is applied to this structure by inspection of the load-plastic work curve of Fig. 4(a). There is a discontinuity in the curve at the point at which bar 3 yields, effectively a curvature of infinity. Thereafter, the structural response is steady-state plastic deformation with no further stress redistribution. Yield of bar 3, or the fully plastic state, is therefore taken as the PWC plastic load.

The beam structure behaves differently from the others in that it never becomes fully plastic. These is also extensive stress redistribution between yield and the gross plastic deformation state for this configuration. The TES, TI, and $\mathrm{PW}$ criteria all give plastic moments for the strain hardening structures close to the limit moment, corresponding to $50 \%$ plastic deformation of the beam cross-section. In limit analysis, $100 \%$ of the beam cross-section experiences (gross) plastic deformation at the limit moment. In this case, the TES, TI and PW criteria do not, therefore, capture the strength enhancing effect of strain hardening. The PWC criterion gives a significantly larger value for plastic moment, corresponding to $90 \%$ plastic deformation of the cross-section of the beam. This signifies gross plastic deformation in the PWC criterion.

Gerdeen's proposal that the ratio of plastic work to elastic work, the $\alpha$ factor, can be used as an indicator of gross plastic deformation was also investigated for the three examples. Gerdeen did not specify a general value for $\alpha$ to be used in DBA and the plots of load against $W_{\mathrm{P}} / W_{\mathrm{E}}$ obtained for the three examples do not indicate a general trend. The $\alpha$ values corresponding to the limit state and fully plastic state (where possible) are shown in Table 2. These indicate that the appropriate value of $\alpha$ to be used in DBA is likely to be problem dependent.

\section{Conclusions}

The investigation indicates that the PWC criterion may prove to be a useful way to determine the plastic load in pressure vessel DBA. The curvature characterises the real physical process, the evolution of plastic deformation with increasing load. Salient points on the curve indicate significant events in the formation of the failure mechanism. The onset of curvature indicates initial yield, the maximum value of curvature indicates a change from elastic to plastic dominated response and the return to low or zero curvature indicates gross plasticity. At this stage in the development criterion no value of curvature or relative curvature is defined as indicating gross plastic deformation and the values specified in the sample analysis are to some extent subjective. The scope of the investigation was limited to three simple structural configurations and two material models. A more extensive investigation of real pressure vessel components, boundary conditions and material models is required to establish if a general and objective formulation of the criterion can be specified. It is proposed to extend the investigation of the PWC criterion to more complex problems in future work.

\section{Acknowledgements}

The authors wish to thank Dr Bobby Hamilton for his help and insight when preparing this paper.

\section{References}

[1] BSI. PD5500:2000 Specification for unfired fusion welded pressure vessels. London: British standards Institution; 2000.

[2] EN 13445. Unfired pressure vessels-Part 3: design. European Committee for Standardisation (CEN); 2002.

[3] ASME. Boiler and pressure vessel code section VIII. New York: American Society for Mechanical Engineers; 2004.

[4] Gerdeen JC. A critical evaluation of plastic behaviour data and a united definition of plastic loads for pressure components. Welding Res Bull 1979;254

[5] Townley CHA, Findlay GE, Goodman AM, Stanley P. Elastic-plastic computations as a basis for design charts for torispherical pressure vessel ends. Proc I Mech E 1971;185(63):869-77.

[6] American Society for Mechanical Engineers, New York, USAASME, Boiler and pressure vessel code; 1971.

[7] American Society for Mechanical Engineers, New York, USAASME, Boiler and pressure vessel code; 1971.

[8] Save M. Experimental verification of plastic limit analysis of torispherical and toriconical heads. Pressure Vessels Piping: Design Analysis 1972;1:382-416 [ASME].

[9] Moffat DG, Hsieh MF, Lynch M. An assessment of ASME III and CEN TC54 methods of determining plastic and limit loads for pressure system components. J Strain Anal 2001;36(3):301-12.

[10] Muscat M, Mackenzie D, Hamilton R. A work criterion for plastic collapse. Int J Pressure Vessel Piping 2003;80:49-58.

[11] Robertson A, Li H, Mackenzie D. Plastic collapse of pipe bends under combined internal pressure and in-plane bending. Int $\mathrm{J}$ Pressure Vessel and Piping 2005;82:407-16.

[12] Lee KS. The influence of attachment size on the limit pressures of defective piping components. $\mathrm{PhD}$ Thesis, The University of Liverpool, UK; 2003.

[13] ANSYs Version 8.0. Canonsberg, PA 15317: ANSYS Inc.; 2003.

[14] Li H. Plastic collapse analysis of pressure vessel components, MPhil Thesis, University of Strathclyde, UK; 2004.

[15] Pro/Engineer, PTC, Needham, MA, USA; 2001.

[16] Lubliner J. Plasticity theory. New York: Macmillan Publishing Company; 1990. 